\title{
Infinitely many solutions for a class of quasilinear elliptic equations with $p$-Laplacian in $\mathbb{R}^{N}$
}

Gao Jia*, Jie Chen and Long-jie Zhang

${ }^{\text {*Correspondence: }}$ gaojia89@163.com; gaojia79@139.com College of Science, University of Shanghai for Science and Technology, Shanghai, 200093, China

\begin{abstract}
In this paper, we study the multiplicity of solutions for a class of quasilinear elliptic equations with $p$-Laplacian in $\mathbb{R}^{N}$. In this case, the functional $J$ is not differentiable. Hence, it is difficult to work under the classical framework of the critical point theory. To overcome this difficulty, we use a nonsmooth critical point theory, which provides the existence of critical points for nondifferentiable functionals.
\end{abstract}

MSC: 35J20; 35J92; 58E05

Keywords: quasilinear elliptic equations; nondifferentiable functional; $p$-Laplacian; multiple solutions

\section{Introduction and main results}

Recently, the multiplicity of solutions for the quasilinear elliptic equations has been studied extensively, and many fruitful results have been obtained. For example, in [1], Shibo Liu considered the existence of multiple nonzero solutions of the Dirichlet boundary value problem

$$
\left\{\begin{array}{l}
-\Delta_{p} u=f(x, u), \quad \text { in } \Omega, \\
u=0, \quad \text { on } \partial \Omega,
\end{array}\right.
$$

where $p>1, \Delta_{p} u=\operatorname{div}\left(|\nabla u|^{p-2} \nabla u\right)$ denotes the $p$-Laplacian operator, $\Omega$ is a bounded domain in $\mathbb{R}^{N}$ with smooth boundary $\partial \Omega$.

Moreover, Aouaoui studied the following quasilinear elliptic equation in [2]:

$$
-\operatorname{div}(A(x, u) \nabla u)+\frac{1}{2} A_{s}^{\prime}(x, u)|\nabla u|^{2}+(b(x)-\lambda) u=f(x, u), \quad \text { in } \mathbb{R}^{N},
$$

and proved the multiplicity of solutions of the problem (1.2) by using the nonsmooth critical point theory. One can refer to $[3,4]$ and [5] for more results.

In this paper, we shall investigate the existence of infinitely many solutions of the following problem

$$
\begin{aligned}
& -\operatorname{div}\left(A(x, u)|\nabla u|^{p-2} \nabla u\right)+\frac{1}{p} A_{s}(x, u)|\nabla u|^{p}+(b(x)-\lambda)|u|^{p-2} u=f(x, u), \\
& \quad \text { in } \mathbb{R}^{N},
\end{aligned}
$$


where $2<p<N, \lambda \in \mathbb{R}$ and $\left.A_{s}(x, u) \equiv \frac{\partial A}{\partial s}(x, s)\right|_{s=u}, b(x)$ is a given continuous function satisfying

$$
b(x) \geq 0 \quad \text { for all } x \in \mathbb{R}^{N} \text { and } \quad \lim _{|x| \rightarrow+\infty} b(x)=+\infty .
$$

In order to determine weak solutions of (1.3) in a suitable functional space $E$, we look for critical points of the functional $J: E \rightarrow \mathbb{R}$ defined by

$$
\begin{aligned}
J(v) & =\frac{1}{p} \int_{\mathbb{R}^{N}} A(x, v)|\nabla v|^{p} d x+\frac{1}{p} \int_{\mathbb{R}^{N}}(b(x)-\lambda)|v|^{p} d x-\int_{\mathbb{R}^{N}} F(x, v) d x, \\
\forall v & \in E
\end{aligned}
$$

where $F(x, \xi)=\int_{0}^{\xi} f(x, t) d t$. Under reasonable assumptions, the functional $J$ is continuous, but not even locally Lipschitz. However, one can see from [4, 6] and [7] that the Gâteauxderivative of $J$ exists in the smooth directions, i.e., it is possible to evaluate

$$
\begin{aligned}
\left\langle J^{\prime}(u), v\right\rangle= & \lim _{t \rightarrow 0} \frac{J(u+t v)-J(u)}{t} \\
= & \int_{\mathbb{R}^{N}} A(x, u)|\nabla u|^{p-2} \nabla u \nabla v d x+\frac{1}{p} \int_{\mathbb{R}^{N}} A_{s}(x, u)|\nabla u|^{p} v d x \\
& +\int_{\mathbb{R}^{N}}(b(x)-\lambda)|u|^{p-2} u v d x-\int_{\mathbb{R}^{N}} f(x, u) v d x
\end{aligned}
$$

for all $u \in E$ and $v \in E \cap L^{\infty}\left(\mathbb{R}^{N}\right)$.

Definition 1.1 A critical point $u$ of the functional $J$ is defined as a function $u \in E$ such that $\left\langle J^{\prime}(u), v\right\rangle=0, \forall v \in E \cap L^{\infty}\left(\mathbb{R}^{N}\right)$, i.e.,

$$
\begin{aligned}
& \int_{\mathbb{R}^{N}} A(x, u)|\nabla u|^{p-2} \nabla u \nabla v d x+\frac{1}{p} \int_{\mathbb{R}^{N}} A_{s}(x, u)|\nabla u|^{p} v d x \\
& \quad+\int_{\mathbb{R}^{N}}(b(x)-\lambda)|u|^{p-2} u v d x-\int_{\mathbb{R}^{N}} f(x, u) v d x=0, \quad \forall v \in E \cap L^{\infty}\left(\mathbb{R}^{N}\right) .
\end{aligned}
$$

Our approach to study (1.3) is based on the nonsmooth critical point theory developed in [8] and [9]. Dealing with this class of problems, the main difficulty is that the associated functional is not differentiable in all directions.

The main goal here is to establish multiplicity of results for (1.3), when $f(x, s)$ is odd and $A(x, s)$ is even in $s$. Such solutions for (1.3) will follow from a version of the symmetric mountain pass theorem due to Ambrosetti and Rabinowitz [10,11]. Compared with problem (1.2) in [2], problem (1.3) is much more difficult, since the discreteness of the spectrum is not guaranteed. Therefore, we only consider the first eigenvalue $\lambda_{1}$.

To state and prove our main result, we consider the following assumptions.

Suppose that $N \geq 3$ and $p^{*}=\frac{N p}{N-p}$.

$\left(\mathrm{H}_{1}\right)$ Let $A(\cdot, \cdot): \mathbb{R}^{N} \times \mathbb{R} \rightarrow \mathbb{R}$ be a function such that

- for each $s \in \mathbb{R}, A(x, s)$ is measurable with respect to $x$;

- for a.e. $x \in \mathbb{R}^{N}, A(x, s)$ is a function of class $C^{1}$ with respect to $s$; 
- there exist $0<\alpha<\beta<+\infty$ such that

$$
\begin{aligned}
& \alpha \leq A(x, s) \leq \beta, \quad \text { a.e. } x \in \mathbb{R}^{N} \text { and } \forall s \in \mathbb{R}, \\
& \left|A_{s}(x, s)\right| \leq \beta, \quad \text { a.e. } x \in \mathbb{R}^{N} \text { and } \forall s \in \mathbb{R} .
\end{aligned}
$$

$\left(\mathrm{H}_{2}\right)$ There exist $\theta>p, 1<\gamma<\frac{\theta}{p}$ and $\alpha_{1}>0$ such that

$$
0 \leq \frac{\gamma}{p} A_{s}(x, s) s \leq\left(\frac{\theta}{p}-\gamma\right) A(x, s)-\alpha_{1}, \quad \text { a.e. } x \in \mathbb{R}^{N} \text { and } \forall s \in \mathbb{R}
$$

$\left(\mathrm{H}_{3}\right)$ Let a Carathéodory function $f(\cdot, \cdot): \mathbb{R}^{N} \times \mathbb{R} \rightarrow \mathbb{R}$ satisfy $f(x, 0)=0$, a.e. $x \in \mathbb{R}^{N}$ and

$$
0<\theta F(x, s) \leq f(x, s) s, \quad \text { a.e. } x \in \mathbb{R}^{N} \text { and } \forall s \neq 0 \text { in } \mathbb{R} \text {, }
$$

where $\theta$ is the same as that in $\left(\mathrm{H}_{2}\right)$.

$\left(\mathrm{H}_{4}\right)$ There exists $p-1 \leq q<p^{*}-1$ such that

$$
|f(x, s)| \leq c_{0}|s|^{q}, \quad \text { a.e. } x \in \mathbb{R}^{N} \text { and } \forall s \in \mathbb{R},
$$

where $c_{0}$ is a positive constant.

Example 1.1 Let $p=3$. The following function satisfies hypotheses $\left(\mathrm{H}_{1}\right)$ and $\left(\mathrm{H}_{2}\right)$

$$
A(x, s)=2\left(\sin \left(|x|^{2}\right)+2\right) \arctan \left(s^{2}\right)+20,
$$

and the corresponding constants are

$$
\alpha=20, \quad \beta=20+3 \pi, \quad \theta=34, \quad \gamma=10, \quad \alpha_{1}=1 .
$$

Example 1.2 The following function satisfies hypotheses $\left(\mathrm{H}_{3}\right)$ and $\left(\mathrm{H}_{4}\right)$

$$
f(x, s)=|s|^{q-1} s, \quad \text { a.e. } x \in \mathbb{R}^{N} \text { and } \forall s \in \mathbb{R} \text {. }
$$

On the other hand, we define the operator $L u=-\Delta_{p} u+b(\cdot) u$. It follows from [12] that the discreteness of the spectrum is not guaranteed. Hence, we only consider the first eigenvalue $\lambda_{1}$, where

$$
\lambda_{1}=\inf \left\{\|u\|^{p} ; u \in E,\|u\|_{L^{p}\left(\mathbb{R}^{N}\right)}=1\right\} .
$$

Next, we can state the main theorem of the paper.

Theorem 1.1 Assume that $A(x, s)$ and $f(x, s)$ satisfy $\left(\mathrm{H}_{1}\right)-\left(\mathrm{H}_{4}\right)$. Moreover, let $A(x,-s)=$ $A(x, s)$ and $f(x,-s)=-f(x, s)$, a.e. $x \in \mathbb{R}^{N}, \forall s \in \mathbb{R}$. If there exists a positive number $\mu$ such that $\lambda \in\left(-\infty, \mu \lambda_{1}\right)$, then problem (1.3) has infinitely many distinct solutions in $E \cap L^{\infty}\left(\mathbb{R}^{N}\right)$, i.e., there exists a sequence $\left\{u_{n}\right\} \subset E \cap L^{\infty}\left(\mathbb{R}^{N}\right)$, satisfying $(1.3)$ and $J\left(u_{n}\right) \rightarrow+\infty$, as $n \rightarrow \infty$. 
To explain our result, we introduce some functional spaces. We define the reflexive Banach space $E$ of all functions $u: \mathbb{R}^{N} \rightarrow \mathbb{R}$ with the norm $\|u\|^{p}=\int_{\mathbb{R}^{N}}\left(|\nabla u|^{p}+b(x)|u|^{p}\right) d x<$ $\infty$.

Such a weighted Sobolev space has been used in many previous papers, see [13] and [14]. Now, we give an important property of the space $E$, which will play an essential role in proving our main results.

Remark 1.1 One can easily deduce $E \hookrightarrow L^{z}\left(\mathbb{R}^{N}\right)$ and $E \hookrightarrow \hookrightarrow L^{z}\left(\mathbb{R}^{N}\right)$ for $p \leq z<p^{*}$. More details can be found in [2].

Throughout this paper, let $\|\cdot\|$ denote the norm of $E$ and $u_{n} \rightarrow u\left(u_{n} \rightarrow u\right)$ means that $u_{n}$ converges strongly (weakly) in corresponding spaces. $\hookrightarrow$ stands for a continuous map, and $\hookrightarrow \hookrightarrow$ means a compact embedding map. $C$ denotes any universal positive constant unless specified.

The paper is organized as follows. In Section 2, we introduce the nonsmooth critical framework and preliminaries to our work. In Section 3, we give some lemmas to prove the main result. Finally, the proof of Theorem 1.1 is presented in Section 4.

\section{Nonsmooth critical framework and preliminaries}

Our results are based on the techniques of nonsmooth critical point theory. In this section, we recall some basic tools from [8] and [9].

Definition 2.1 Let $(X, d)$ be a metric space, let $I: X \rightarrow \mathbb{R}$ be a continuous functional and $u \in X$. We denote by $|d I|(u)$ the supremum of the $\sigma$ 's in $[0,+\infty)$ such that there exist $\delta>0$ and a continuous map $H: B(u, \delta) \times[0, \delta] \rightarrow X$, satisfying

$$
d(H(v, t), v) \leq t \quad \text { and } \quad I(H(v, t)) \leq I(v)-\sigma t, \quad(v, t) \in B(u, \delta) \times[0, \delta] .
$$

The extended real number $|d I|(u)$ is called the weak slope of $I$ at $u$.

Note that the notion above was independently introduced in [15], as well.

Definition 2.2 Let $(X, d)$ be a metric space, let $I: X \rightarrow \mathbb{R}$ be a continuous functional and $c \in \mathbb{R}$. We say that $I$ satisfies $(P-S)_{c}$, i.e., the Palais-Smale condition at level $c$, if every sequence $\left\{u_{n}\right\}$ in $\mathrm{X}$ with $|d I|\left(u_{n}\right) \rightarrow 0$ and $I\left(u_{n}\right) \rightarrow c$ admits a strongly convergent subsequence.

In order to treat the Palais-Smale condition, we need to introduce an auxiliary notion.

Definition 2.3 Let $c$ be a real number. We say that functional $I$ satisfies the concrete Palais-Smale condition at level $c\left((C-P-S)_{c}\right.$ for short) if every sequence $\left\{u_{n}\right\} \subset E$ satisfying

$$
\lim _{n \rightarrow+\infty} I\left(u_{n}\right)=c \quad \text { and } \quad\left|\left\langle I^{\prime}\left(u_{n}\right), v\right\rangle\right| \leq \epsilon_{n}\|v\|, \quad \forall v \in E \cap L^{\infty}\left(\mathbb{R}^{N}\right)
$$

possesses a strongly convergent subsequence in $E$, where $\left\{\epsilon_{n}\right\}$ is some real number converging to zero. 
Remark 2.1 Under assumptions $\left(\mathrm{H}_{1}\right)-\left(\mathrm{H}_{4}\right)$, if the functional $J$ satisfies $(1.4)$, then $J$ is continuous, and for every $u \in E$ we have

$$
|d J|(u) \geq \sup \left\{\left\langle J^{\prime}(u), v\right\rangle ; v \in E \cap L^{\infty}\left(\mathbb{R}^{N}\right),\|v\| \leq 1\right\},
$$

where $|d J|(u)$ denotes the weak slope of $J$ at $u$.

Remark 2.2 Let $c$ be a real number. If $J$ satisfies $(C-P-S)_{c}$, then $J$ satisfies $(P-S)_{c}$.

Proof Let $\left\{u_{n}\right\} \subset E$ be a sequence such that

$$
\lim _{n \rightarrow+\infty}|d J|\left(u_{n}\right)=0 \text { and } \lim _{n \rightarrow+\infty} J\left(u_{n}\right)=c .
$$

Note that for $v \subset E \cap L^{\infty}\left(\mathbb{R}^{N}\right)$,

$$
\left|\left\langle J^{\prime}\left(u_{n}\right), v\right\rangle\right| \leq\left\|J^{\prime}\left(u_{n}\right)\right\|\|v\|=\sup \left\{\left\langle J^{\prime}\left(u_{n}\right), v\right\rangle,\|v\| \leq 1\right\}\|v\| .
$$

By Remark 2.1, we have $|d J|\left(u_{n}\right) \geq \sup \left\{\left\langle J^{\prime}\left(u_{n}\right), v\right\rangle,\|v\| \leq 1\right\}$. Taking $\epsilon_{n}=\sup \left\{\left\langle J^{\prime}\left(u_{n}\right), v\right\rangle,\|v\| \leq\right.$ $1\}$, the conclusion follows.

\section{Basic lemmas}

To derive our main theorem, we need the following lemmas. The first lemma is the version of the Ambrosetti-Rabinowitz mountain pass lemma [10,11] and [16].

Lemma 3.1 Let $X$ be an infinite-dimensional Banach space, and let $I: X \rightarrow \mathbb{R}$ be a continuous even functional satisfying $(P-S)_{c}$ for every $c \in \mathbb{R}$. Assume that

(i) there exist $\varrho>0, \alpha>I(0)$ and a subspace $V \subset X$ of finite codimension such that

$$
\forall u \in\{V:\|u\|=\varrho\} \quad \Rightarrow \quad I(u) \geq \alpha
$$

(ii) for every finite-dimensional subspace $W \subset X$, there exists $\mathbf{R}>0$ such that

$$
\forall u \in\{W:\|u\|=\mathbf{R}\} \quad \Rightarrow \quad I(u) \leq I(0) .
$$

Then there exists a sequence $\left\{c_{h}\right\}$ of critical values of I with $c_{h} \rightarrow \infty$.

Lemma 3.2 If $u \in E$ is a critical point of $J$, then $u \in L^{\infty}\left(\mathbb{R}^{N}\right)$.

Proof For $r>1, M>0$, consider the real functions $T_{r}, U_{M}$ and $W_{M}$ defined in $\mathbb{R}$ by

$$
T_{r}(s)= \begin{cases}s-r, & \text { if } s>r, \\ 0, & \text { if }-r \leq s \leq r, \\ s+r, & \text { if } s<-r,\end{cases}
$$


$U_{M}=\min \left(T_{r}(s), M\right)$ and $W_{M}=\max \left(T_{r}(s),-M\right)$. Denoting $s^{+}=\max (s, 0)$ and $s^{-}=\min (s, 0)$, we can take $v=U_{M}\left(u^{+}\right) \in E \cap L^{\infty}\left(\mathbb{R}^{N}\right)$ as a test function in (1.5). Therefore,

$$
\begin{aligned}
& \int_{\mathbb{R}^{N}} A(x, u)|\nabla u|^{p-2} \nabla u \nabla\left(U_{M}\left(u^{+}\right)\right) d x+\frac{1}{p} \int_{\mathbb{R}^{N}} A_{s}(x, u)|\nabla u|^{p} U_{M}\left(u^{+}\right) d x \\
& +\int_{\mathbb{R}^{N}}(b(x)-\lambda)|u|^{p-2} u U_{M}\left(u^{+}\right) d x=\int_{\mathbb{R}^{N}} f(x, u) U_{M}\left(u^{+}\right) d x
\end{aligned}
$$

Noting that $u^{+} \cdot U_{M}\left(u^{+}\right) \geq 0$ and $A_{s}\left(x, u^{+}\right) U_{M}\left(u^{+}\right) \geq 0$, we get

$$
\begin{aligned}
& \int_{\mathbb{R}^{N}} A\left(x, u^{+}\right)\left|\nabla u^{+}\right|^{p-2} \nabla u^{+} \nabla\left(U_{M}\left(u^{+}\right)\right) d x \\
& \quad \leq|\lambda| \int_{\mathbb{R}^{N}}\left|u^{+}\right|^{p-2} u^{+}\left|U_{M}\left(u^{+}\right)\right| d x+\int_{\mathbb{R}^{N}}\left|f\left(x, u^{+}\right)\right|\left|U_{M}\left(u^{+}\right)\right| d x .
\end{aligned}
$$

From (1.10) and the fact $\left|U_{M}\left(u^{+}\right)\right| \leq u^{+}$we deduce

$$
\int_{\left\{u^{+}>r\right\}} A\left(x, u^{+}\right)\left|\nabla u^{+}\right|^{p-2} \nabla u^{+} \nabla\left(U_{M}\left(u^{+}\right)\right) d x \leq C \int_{\left\{u^{+}>r\right\}}\left(u^{+}\right)^{q+1} d x .
$$

Since $U_{M}\left(u^{+}\right) \rightarrow T_{r}\left(u^{+}\right)$a.e. in $\mathbb{R}^{N}$ and $U_{M}\left(u^{+}\right) \rightarrow T_{r}\left(u^{+}\right)$in $E$ as $M \rightarrow+\infty$. It follows from $M \rightarrow+\infty$ that

$$
\int_{\left\{u^{+}>r\right\}} A\left(x, u^{+}\right)\left|\nabla u^{+}\right|^{p} d x \leq C \int_{\left\{u^{+}>r\right\}}\left(u^{+}\right)^{q+1} d x .
$$

Denote $\Omega_{r}^{+}=\left\{x \in \mathbb{R}^{N}, u^{+}(x)>r\right\}$. If $\operatorname{mes}\left(\Omega_{r}^{+}\right)=0$, then the result is true. In the following discussion, $\operatorname{mes}\left(\Omega_{r}^{+}\right) \neq 0$ is assumed. By (1.6), we obtain

$$
\int_{\Omega_{r}^{+}}\left|\nabla u^{+}\right|^{p} d x \leq C \int_{\Omega_{r}^{+}}\left(u^{+}-r\right)^{q+1} d x+C r^{q+1} \operatorname{mes}\left(\Omega_{r}^{+}\right) .
$$

Note that $\left(\int_{\Omega_{r}^{+}}\left(u^{+}-r\right)^{q+1} d x\right)^{1-\frac{p}{q+1}} \leq C$, then we can get

$$
\int_{\Omega_{r}^{+}}\left(u^{+}-r\right)^{q+1} d x \leq C\left(\int_{\Omega_{r}^{+}}\left(u^{+}-r\right)^{q+1} d x\right)^{\frac{p}{q+1}}
$$

On the other hand, we have

$$
\int_{\Omega_{r}^{+}} r^{p^{*}} d x \leq \int_{\Omega_{r}^{+}}|u|^{p^{*}} d x=C,
$$

which implies that

$$
r^{p^{*}} \leq \frac{C}{\operatorname{mes}\left(\Omega_{r}^{+}\right)}
$$

Eventually, one can deduce from (3.2)-(3.4) that

$$
\int_{\Omega_{r}^{+}}\left|\nabla u^{+}\right|^{p} d x \leq C\left(\int_{\Omega_{r}^{+}}\left(u^{+}-r\right)^{q+1} d x\right)^{\frac{p}{q+1}}+C r^{p} \operatorname{mes}\left(\Omega_{r}^{+}\right)^{1-\frac{q+1}{p^{*}}+\frac{p}{p^{*}}}, \quad \forall r>1 .
$$


By Theorem 5.2 of [17], we get that $u^{+} \in L^{\infty}\left(\mathbb{R}^{N}\right)$. Replacing $U_{M}\left(u^{+}\right)$by $W\left(u^{-}\right)$, we can similarly prove that $u^{-} \in L^{\infty}\left(\mathbb{R}^{N}\right)$. We conclude that $u \in L^{\infty}\left(\mathbb{R}^{N}\right)$, and the proof of Lemma 3.2 is completed.

Lemma 3.3 Let $\left\{u_{n}\right\}$ be a bounded sequence in $E$ with

$$
\left\langle J^{\prime}\left(u_{n}\right), v\right\rangle \leq \epsilon_{n}\|v\|, \quad \forall v \in E \cap L^{\infty}\left(\mathbb{R}^{N}\right)
$$

where $\left\{\epsilon_{n}\right\}$ is a sequence of real numbers converging to zero. Then there exists $u \in E$ such that $\nabla u_{n} \rightarrow \nabla u$ a.e. in $\mathbb{R}^{N}$ and, up to a subsequence, $\left\{u_{n}\right\}$ is weakly convergent to $u$ in $E$. Moreover, we have

$$
\left\langle J^{\prime}(u), v\right\rangle=0, \quad \forall v \in E \cap L^{\infty}\left(\mathbb{R}^{N}\right)
$$

i.e., $u$ is a critical point of J.

Proof Since $\left\{u_{n}\right\}$ is bounded in $E$, and there is a $u \in E$ (see [18]) such that, up to a subsequence,

$$
u_{n} \rightarrow u \quad \text { in } E, \quad u_{n} \rightarrow u \quad \text { in } L^{p}\left(\mathbb{R}^{N}\right), \quad u_{n} \rightarrow u \quad \text { a.e. in } \mathbb{R}^{N}
$$

Moreover, since $\left\{u_{n}\right\}$ satisfies (3.6), by Theorem 2.1 of [19], we have, up to a further subsequence, $\nabla u_{n} \rightarrow \nabla u$ a.e. in $\mathbb{R}^{N}$.

We will use the device of [20]. We consider the test functions

$$
v_{n}=\varphi \exp \left\{-M u_{n}^{+}\right\}
$$

where $\varphi \in E \cap L^{\infty}\left(\mathbb{R}^{N}\right), \varphi \geq 0$ and $M>0$. According to (1.6) and (1.7), we have

$$
M A\left(x, u_{n}\right) \geq \frac{1}{p}\left|A_{s}\left(x, u_{n}\right)\right|
$$

Since (3.6) holds by density for every $v \in E \cap L^{\infty}\left(\mathbb{R}^{N}\right)$, we can put $v=v_{n}$ in (3.6) and obtain that

$$
\begin{aligned}
& \int_{\mathbb{R}^{N}} A\left(x, u_{n}\right)\left|\nabla u_{n}\right|^{p-2} \nabla u_{n} \nabla \varphi \exp \left\{-M u_{n}^{+}\right\} d x \\
& \quad+\int_{\mathbb{R}^{N}}\left(\frac{1}{p} A_{s}\left(x, u_{n}\right)\left|\nabla u_{n}\right|^{p}-M A\left(x, u_{n}\right)\left|\nabla u_{n}\right|^{p-2} \nabla u_{n} \nabla u_{n}^{+}\right) \varphi \exp \left\{-M u_{n}^{+}\right\} d x \\
& \quad+\int_{\mathbb{R}^{N}}(b(x)-\lambda)\left|u_{n}\right|^{p-2} u_{n} \varphi \exp \left\{-M u_{n}^{+}\right\} d x \\
& \quad-\int_{\mathbb{R}^{N}} f\left(x, u_{n}\right) \varphi \exp \left\{-M u_{n}^{+}\right\} d x \geq-\epsilon_{n}\|v\|
\end{aligned}
$$

On the other hand, note that

$$
\int_{\mathbb{R}^{N}}\left(\frac{1}{p} A_{s}\left(x, u_{n}\right)\left|\nabla u_{n}\right|^{p}-M A\left(x, u_{n}\right)\left|\nabla u_{n}\right|^{p-2} \nabla u_{n} \nabla u_{n}^{+}\right) \varphi \exp \left\{-M u_{n}^{+}\right\} d x \leq 0 .
$$


One can deduce from (3.10) and Fatou's lemma that

$$
\begin{array}{rl}
\int_{\mathbb{R}^{N}} & A(x, u)|\nabla u|^{p-2} \nabla u \nabla \varphi \exp \left\{-M u^{+}\right\} d x \\
& +\int_{\mathbb{R}^{N}}\left(\frac{1}{p} A_{s}(x, u)|\nabla u|^{p}-M A(x, u)|\nabla u|^{p-2} \nabla u \nabla u^{+}\right) \varphi \exp \left\{-M u^{+}\right\} d x \\
& +\int_{\mathbb{R}^{N}}(b(x)-\lambda)|u|^{p-2} u \varphi \exp \left\{-M u^{+}\right\} d x-\int_{\mathbb{R}^{N}} f(x, u) \varphi \exp \left\{-M u^{+}\right\} d x \\
\geq & 0
\end{array}
$$

We consider the test functions $\varphi_{n}=\varphi g\left(\frac{u}{n}\right) \exp \left\{M u^{+}\right\}$with $\varphi \in E \cap L^{\infty}\left(\mathbb{R}^{N}\right), \varphi \geq 0$ and $g: \mathbb{R} \rightarrow \mathbb{R}, g \in C^{1}(\mathbb{R}), 0 \leq g \leq 1$,

$$
g=1 \quad \text { on }\left[-\frac{1}{p}, \frac{1}{p}\right], \quad g=0 \quad \text { on }[-\infty,-1] \cup[1, \infty] \text {. }
$$

This together with (3.11) can prove that

$$
\begin{gathered}
\int_{\mathbb{R}^{N}} A(x, u)|\nabla u|^{p-2} \nabla u \nabla \varphi d x+\frac{1}{p} \int_{\mathbb{R}^{N}} A_{s}(x, u)|\nabla u|^{p} \varphi d x \\
+\int_{\mathbb{R}^{N}}(b(x)-\lambda)|u|^{p-2} u \varphi d x-\int_{\mathbb{R}^{N}} f(x, u) \varphi d x \geq 0, \\
\forall \varphi \in E \cap L^{\infty}\left(\mathbb{R}^{N}\right), \text { as } n \rightarrow \infty .
\end{gathered}
$$

In a similar way, by considering the test functions $v_{n}=\varphi \exp \left\{M u_{n}^{-}\right\}$, it is possible to prove that

$$
\begin{gathered}
\int_{\mathbb{R}^{N}} A(x, u)|\nabla u|^{p-2} \nabla u \nabla \varphi d x+\frac{1}{p} \int_{\mathbb{R}^{N}} A_{s}(x, u)|\nabla u|^{p} \varphi d x \\
+\int_{\mathbb{R}^{N}}(b(x)-\lambda)|u|^{p-2} u \varphi d x-\int_{\mathbb{R}^{N}} f(x, u) \varphi d x \leq 0, \\
\forall \varphi \in E \cap L^{\infty}\left(\mathbb{R}^{N}\right), \text { as } n \rightarrow \infty .
\end{gathered}
$$

From (3.12) and (3.13), it follows that

$$
\begin{aligned}
& \int_{\mathbb{R}^{N}} A(x, u)|\nabla u|^{p-2} \nabla u \nabla \varphi d x+\frac{1}{p} \int_{\mathbb{R}^{N}} A_{s}(x, u)|\nabla u|^{p} \varphi d x \\
& +\int_{\mathbb{R}^{N}}(b(x)-\lambda)|u|^{p-2} u \varphi d x-\int_{\mathbb{R}^{N}} f(x, u) \varphi d x=0, \quad \forall \varphi \in E \cap L^{\infty}\left(\mathbb{R}^{N}\right) .
\end{aligned}
$$

Finally, we can deduce (3.7) from (3.14).

Remark 3.1 (see [21]) Let $\left\{u_{n}\right\}$ be a sequence in $E$ satisfying (3.6). Then $-\lambda\left|u_{n}\right|^{p}-$ $f\left(x, u_{n}\right) u_{n} \in L^{1}\left(\mathbb{R}^{N}\right)$ and

$$
\begin{aligned}
& \left|\int_{\mathbb{R}^{N}}\left(A\left(x, u_{n}\right)\left|\nabla u_{n}\right|^{p}+\frac{1}{p} A_{s}\left(x, u_{n}\right) u_{n}\left|\nabla u_{n}\right|^{p}+(b(x)-\lambda)\left|u_{n}\right|^{p}-f\left(x, u_{n}\right) u_{n}\right) d x\right| \\
& \quad \leq \epsilon_{n}\left\|u_{n}\right\|, \quad \forall n \in \mathbb{N} .
\end{aligned}
$$


In the following lemma, we will prove the boundedness of a $(C-P-S)_{c}$ sequence $\left\{u_{n}\right\} \subset$ $E$ under (1.6), (1.8) and (1.9).

Lemma 3.4 Let $c \in \mathbb{R}$ and $\left\{u_{n}\right\}$ be a sequence in E satisfying (3.6) and

$$
\lim _{n \rightarrow+\infty} J\left(u_{n}\right)=c
$$

Then $\left\{u_{n}\right\}$ is bounded in E.

Proof Calculating $\theta J\left(u_{n}\right)-\gamma\left\langle J^{\prime}\left(u_{n}\right), u_{n}\right\rangle$, from (3.15) and (3.16), we obtain

$$
\begin{aligned}
& \left(\frac{\theta}{p}-\gamma\right) \int_{\mathbb{R}^{N}} A\left(x, u_{n}\right)\left|\nabla u_{n}\right|^{p} d x-\frac{\gamma}{p} \int_{\mathbb{R}^{N}} A_{s}\left(x, u_{n}\right)\left|\nabla u_{n}\right|^{p} u_{n} d x \\
& \quad+\left(\frac{\theta}{p}-\gamma\right) \int_{\mathbb{R}^{N}}(b(x)-\lambda)\left|u_{n}\right|^{p} d x+\int_{\mathbb{R}^{N}}\left(\gamma f\left(x, u_{n}\right) u_{n}-\theta F\left(x, u_{n}\right)\right) d x \\
& \leq C\left(1+\left\|u_{n}\right\|\right), \quad \forall n \in \mathbb{N} .
\end{aligned}
$$

From (1.8) and (1.9), it follows that

$$
\begin{aligned}
& \alpha_{1} \int_{\mathbb{R}^{N}}\left|\nabla u_{n}\right|^{p} d x+\left(\frac{\theta}{p}-\gamma\right) \int_{\mathbb{R}^{N}}(b(x)-\lambda)\left|u_{n}\right|^{p} d x+\theta(\gamma-1) \int_{\mathbb{R}^{N}} F\left(x, u_{n}\right) d x \\
& \quad \leq C\left(1+\left\|u_{n}\right\|\right), \quad \forall n \in \mathbb{N} .
\end{aligned}
$$

Moreover, there exist $M>0$ and $C_{1}(\lambda)>0$ such that

$$
\left(\frac{\theta}{p}-\gamma\right) \int_{\mathbb{R}^{N}}(b(x)-\lambda)\left|u_{n}\right|^{p} d x \geq\left(\frac{\theta}{p}-\gamma\right) \int_{\mathbb{R}^{N}} \frac{b(x)}{2}\left|u_{n}\right|^{p} d x-C_{1} \int_{\{|x|<M\}}\left|u_{n}\right|^{p} d x
$$

Therefore, denoting $D_{M}=\left\{x \in \mathbb{R}^{N},|x|<M\right\}$, we obtain from (3.17) that

$$
\begin{aligned}
& \alpha_{1} \int_{\mathbb{R}^{N}}\left|\nabla u_{n}\right|^{p} d x+\left(\frac{\theta}{p}-\gamma\right) \int_{\mathbb{R}^{N}} \frac{b(x)}{2}\left|u_{n}\right|^{p} d x+\theta(\gamma-1) \int_{\mathbb{R}^{N}} F\left(x, u_{n}\right) d x \\
& \leq C\left(1+\left\|u_{n}\right\|\right)+C_{1}\left\|u_{n}\right\|_{L^{p}\left(D_{M}\right)}^{p}, \quad \forall n \in N .
\end{aligned}
$$

By virtue of hypothesis $\left(\mathrm{H}_{3}\right)$, we know that there exist $a_{0}>0$ and $b_{0}>0$ such that

$$
F(x, s) \geq a_{0}|s|^{\theta}-b_{0}, \quad \text { a.e. } x \in D_{M} \text { and } \forall s \in \mathbb{R} \text {. }
$$

From (3.18) and (3.19), it follows that

$$
\begin{aligned}
& \min \left(\alpha_{1}, \frac{1}{2}\left(\frac{\theta}{p}-\gamma\right)\right)\left\|u_{n}\right\|^{p}+\theta(\gamma-1) a_{0}\left\|u_{n}\right\|_{L^{\theta}\left(D_{M}\right)}^{\theta} \\
& \leq C\left(1+\left\|u_{n}\right\|\right)+b_{0} \theta(\gamma-1) \operatorname{mes}\left(D_{M}\right)+C_{1}\left\|u_{n}\right\|_{L^{p}\left(D_{M}\right)}^{p}
\end{aligned}
$$

On the other hand, by Hölder's inequality and Young's inequality, for all $\epsilon>0$, there exists $C_{\epsilon}>0$ such that

$$
C_{1}\left\|u_{n}\right\|_{L^{p}\left(D_{M}\right)}^{p} \leq C\left\|u_{n}\right\|_{L^{\theta}\left(D_{M}\right)}^{p} \leq C_{\epsilon}+\epsilon\left\|u_{n}\right\|_{L^{\theta}\left(D_{M}\right)}^{\theta}
$$


Using (3.20) and (3.21), we get

$$
\begin{aligned}
& \min \left(\alpha_{1}, \frac{1}{2}\left(\frac{\theta}{p}-\gamma\right)\right)\left\|u_{n}\right\|^{p} \\
& \quad \leq C\left(1+\left\|u_{n}\right\|\right)+b_{0} \theta(\gamma-1) \operatorname{mes}\left(D_{M}\right)+C_{\epsilon}+\left(\epsilon-\theta(\gamma-1) a_{0}\right)\left\|u_{n}\right\|_{L^{\theta}\left(D_{M}\right)}^{\theta} .
\end{aligned}
$$

Choosing $0<\epsilon<\theta(\gamma-1) a_{0}$ in (3.22), we find that $\left\{u_{n}\right\}$ is bounded in $E$.

Lemma 3.5 Let $\left\{u_{n}\right\}$ be the same as that in Lemma 3.3. Then $\left\{u_{n}\right\}$, up to a subsequence, converges strongly to $u$ in $E$.

Proof By Lemma 3.3, we know that $u$ is a critical point of the functional $J$. Then, from Lemma 3.2, we get $u \in L^{\infty}\left(\mathbb{R}^{N}\right)$. Therefore, taking $v=u$ as a test function in (3.7), we get

$$
\begin{aligned}
& \int_{\mathbb{R}^{N}} A(x, u)|\nabla u|^{p} d x+\frac{1}{p} \int_{\mathbb{R}^{N}} A_{s}(x, u) u|\nabla u|^{p} d x+\int_{\mathbb{R}^{N}}(b(x)-\lambda)|u|^{p} d x \\
& =\int_{\mathbb{R}^{N}} f(x, u) u d x .
\end{aligned}
$$

By virtue of $\left\{u_{n}\right\}$ is bounded in $E$, we can assume that there exists $u \in E$ satisfying

$$
u_{n} \rightarrow u \quad \text { in } E \quad \text { and } \quad u_{n} \rightarrow u \quad \text { in } L^{p}\left(\mathbb{R}^{N}\right) \quad \text { and } \quad u_{n} \rightarrow u \quad \text { a.e. } x \in \mathbb{R}^{N} \text {. }
$$

By Lemma 3.3, $\nabla u_{n} \rightarrow \nabla u$ a.e. in $\mathbb{R}^{N}$. Then by Fatou's lemma, we have

$$
\int_{\mathbb{R}^{N}} A_{s}(x, u) u|\nabla u|^{p} d x \leq \liminf _{n \rightarrow+\infty} \int_{\mathbb{R}^{N}} A_{s}\left(x, u_{n}\right) u_{n}\left|\nabla u_{n}\right|^{p} d x
$$

Moreover, by $E \hookrightarrow \hookrightarrow L^{p}\left(\mathbb{R}^{N}\right)$ and $E \hookrightarrow \hookrightarrow L^{q+1}\left(\mathbb{R}^{N}\right)$, we get

$$
\begin{aligned}
& \lim _{n \rightarrow+\infty} \int_{\mathbb{R}^{N}} f\left(x, u_{n}\right) u_{n} d x=\int_{\mathbb{R}^{N}} f(x, u) u d x, \\
& \lim _{n \rightarrow+\infty} \int_{\mathbb{R}^{N}}\left|u_{n}\right|^{p} d x=\int_{\mathbb{R}^{N}}|u|^{p} d x .
\end{aligned}
$$

By using (3.23)-(3.26) and passing to limit in (3.15), we obtain

$$
\begin{gathered}
\limsup _{n \rightarrow+\infty}\left(\int_{\mathbb{R}^{N}} A\left(x, u_{n}\right)\left|\nabla u_{n}\right|^{p} d x+\int_{\mathbb{R}^{N}} b(x)\left|u_{n}\right|^{p} d x\right) \\
\leq \int_{\mathbb{R}^{N}} A(x, u)|\nabla u|^{p} d x+\int_{\mathbb{R}^{N}} b(x)|u|^{p} d x .
\end{gathered}
$$

On the other hand, by Lebesgue's dominated convergence theorem and the weak convergence of $u_{n}$ to $u$ in $E$, we get

$$
\begin{aligned}
& \lim _{n \rightarrow+\infty} \int_{\mathbb{R}^{N}} A\left(x, u_{n}\right) \nabla u_{n}|\nabla u|^{p-2} \nabla u d x=\int_{\mathbb{R}^{N}} A(x, u)|\nabla u|^{p} d x, \\
& \lim _{n \rightarrow+\infty} \int_{\mathbb{R}^{N}} A\left(x, u_{n}\right)|\nabla u|^{p} d x=\int_{\mathbb{R}^{N}} A(x, u)|\nabla u|^{p} d x,
\end{aligned}
$$




$$
\lim _{n \rightarrow+\infty} \int_{\mathbb{R}^{N}} b(x)|u|^{p-2} u u_{n} d x=\int_{\mathbb{R}^{N}} b(x)|u|^{p} d x
$$

Moreover, since $A\left(x, u_{n}\right)\left|\nabla u_{n}\right|^{p-2} \nabla u_{n}$ and $b(x)^{\frac{p-1}{p}}\left|u_{n}\right|^{p-2} u_{n}$ are bounded in $L^{\frac{p}{p-1}}\left(\mathbb{R}^{N}\right)$, then we have

$$
\begin{aligned}
& A\left(x, u_{n}\right)\left|\nabla u_{n}\right|^{p-2} \nabla u_{n} \rightarrow A(x, u)|\nabla u|^{p-2} \nabla u \quad \text { in } L^{\frac{p}{p-1}}\left(\mathbb{R}^{N}\right) \\
& b(x)^{\frac{p-1}{p}}\left|u_{n}\right|^{p-2} u_{n} \rightarrow b(x)^{\frac{p-1}{p}}|u|^{p-2} u \quad \text { in } L^{\frac{p}{p-1}}\left(\mathbb{R}^{N}\right) .
\end{aligned}
$$

Therefore, from the definition of weak convergence, we obtain

$$
\begin{aligned}
& \lim _{n \rightarrow+\infty} \int_{\mathbb{R}^{N}} A\left(x, u_{n}\right)\left|\nabla u_{n}\right|^{p-2} \nabla u_{n} \nabla u d x=\int_{\mathbb{R}^{N}} A(x, u)|\nabla u|^{p} d x, \\
& \lim _{n \rightarrow+\infty} \int_{\mathbb{R}^{N}} b(x)\left|u_{n}\right|^{p-2} u_{n} u d x=\int_{\mathbb{R}^{N}} b(x)|u|^{p} d x .
\end{aligned}
$$

Combining (3.27)-(3.32), it follows that

$$
\begin{aligned}
& \limsup _{n \rightarrow+\infty}\left(\int_{\mathbb{R}^{N}} A\left(x, u_{n}\right)\left(\left|\nabla u_{n}\right|^{p-2} \nabla u_{n}-|\nabla u|^{p-2} \nabla u\right)\left(\nabla u_{n}-\nabla u\right) d x\right. \\
& \left.\quad+\int_{\mathbb{R}^{N}} b(x)\left(\left|u_{n}\right|^{p-2} u_{n}-|u|^{p-2} u\right)\left(u_{n}-u\right) d x\right) \leq 0
\end{aligned}
$$

It is well known that the following inequality

$$
\left(|\xi|^{t-2} \xi-|\eta|^{t-2} \eta\right)(\xi-\eta)>0
$$

holds for any $t>1, \xi, \eta \in \mathbb{R}^{N}$ and $\xi \neq \eta$. Therefore,

$$
\limsup _{n \rightarrow+\infty}\left(\int_{\mathbb{R}^{N}} A\left(x, u_{n}\right)\left|\nabla u_{n}-\nabla u\right|^{p} d x+\int_{\mathbb{R}^{N}} b(x)\left|u_{n}-u\right|^{p} d x\right) \leq 0
$$

According to (1.6), we conclude that $\left\{u_{n}\right\}$ converges strongly to $u$ in $E$.

Lemma 3.6 For every real number $c$, the functional J satisfies $(C-P-S)_{c}$.

Proof Let $\left\{u_{n}\right\}$ be a sequence in $E$ satisfying (3.6) and (3.16). By Lemma 3.4, $\left\{u_{n}\right\}$ is bounded in $E$. Therefore, the conclusion can be deduced from Lemma 3.5.

\section{Proof of Theorem 1.1}

It is easy to check that the functional $J$ is continuous and even. Moreover, by Remark 2.2 and Lemma 3.6, $J$ satisfies $(P-S)_{c}$ for every $c \in \mathbb{R}$.

On the other hand, from (1.4), (1.6), (1.9) and (1.10), for $u \in E$, we have

$$
J(u) \geq \frac{\min (1, \alpha)}{p}\|u\|^{p}-\frac{\lambda}{p} \int_{\mathbb{R}^{N}}|u|^{p} d x-C\|u\|^{q+1}
$$

We discuss (4.1) in the following two cases: 
In case $\lambda \leq 0$, we get

$$
J(u) \geq \frac{\min (1, \alpha)}{p}\|u\|^{p}-C\|u\|^{q+1} .
$$

In case $\lambda>0$, by the definition of $\lambda_{1}$, we get

$$
J(u) \geq \frac{1}{p}\left(\min (1, \alpha)-\frac{\lambda}{\lambda_{1}}\right)\|u\|^{p}-C\|u\|^{q+1},
$$

i.e., $\mu=\min (1, \alpha)$. Therefore, if $\lambda$ satisfies $\lambda<\min (1, \alpha) \lambda_{1}$, there exist $\varrho>0$ small enough and $\delta>0$ such that

$$
J(u) \geq \delta \quad \text { for }\|u\|=\varrho .
$$

Hence, condition (i) of Lemma 3.1 holds with $V=E$.

Now we consider a finite-dimensional subspace $W$ of $E$. Let $u \in W$ and $J(u) \geq 0$. From (1.6), we have

$$
0 \leq J(u) \leq \max (1, \beta)\|u\|^{p}-\frac{\lambda}{p}\|u\|_{L^{p}\left(\mathbb{R}^{N}\right)}^{p}-\int_{\mathbb{R}^{N}} F(x, u) d x .
$$

By virtue of (1.9) and (1.10), we know that there exist $z(x) \in L^{\infty}\left(\mathbb{R}^{N}\right)$, satisfying $z(x)>0$ a.e. $x \in \mathbb{R}^{N}$ and a positive constant $C_{2}$ such that

$$
F(x, s) \geq z(x)|s|^{\theta}-C_{2}|s|^{p}, \quad \text { a.e. } x \in \mathbb{R}^{N} \text { and } \forall s \in \mathbb{R} .
$$

Combining (4.2)-(4.3), we have

$$
\max (1, \beta)\|u\|^{p} \geq \int_{\mathbb{R}^{N}} z(x)|u|^{\theta} d x-C_{2} \int_{\mathbb{R}^{N}}|u|^{p} d x .
$$

Since $W$ is finite-dimensional, then all norms of $W$ are equivalent. From (4.4), there exists $C_{3}>0$ such that

$$
\|u\|^{\theta} \leq C_{3}\|u\|^{p}
$$

In view of $\theta>p$, we deduce that the set $\{u \in W, J(u) \geq 0\}$ is bounded in $E$ and condition (ii) of Lemma 3.1 holds. By Lemma 3.1, the conclusion follows.

\section{Competing interests}

The authors declare that they have no competing interests.

Authors' contributions

We declare that all authors collaborated and dedicated the same amount of time in order to perform this article.

\section{Acknowledgements}

The authors express their sincere thanks to the referees for their valuable criticism of the manuscript and for helpful suggestions. This work has been supported by the Natural Science Foundation of China (No. 11171220) and Shanghai Leading Academic Discipline Project (XTKX2012). 


\section{References}

1. Liu, SB: Multiplicity results for coercive $p$-Laplacian equations. J. Math. Anal. Appl. 316, 229-236 (2006)

2. Aouaoui, S: Multiplicity of solutions for quasilinear elliptic equations in $\mathbb{R}^{N}$. J. Math. Anal. Appl. 370(2), 639-648 (2010)

3. Alves, $\mathrm{CO}$, Carrião, $\mathrm{PC}$, Miyagaki, OH: Existence and multiplicity results for a class of resonant quasilinear elliptic problems on $\mathbb{R}^{N}$. Nonlinear Anal. 39, 99-110 (2000)

4. Canino, A: Multiplicity of solutions for quasilinear elliptic equations. Topol. Methods Nonlinear Anal. 6, 357-370 (1995)

5. Squassina, M: Existence of multiple solutions for quasilinear diagonal elliptic systems. Electron. J. Differ. Equ. 1999, 1-12 (1999)

6. Arcoya, D, Boccardo, L: Critical points for multiple integrals of the calculus of variations. Arch. Ration. Mech. Anal. 134, 249-274 (1996)

7. Arcoya, D, Boccardo, L: Some remarks on critical point theory for nondifferentiable functionals. NoDEA Nonlinear Differ. Equ. Appl. 6, 79-100 (1999)

8. Corvellec, JN, Degiovanni, M, Marzocchi, M: Deformation properties of continuous functionals and critical point theory. Topol. Methods Nonlinear Anal. 1, 151-171 (1993)

9. Degiovanni, M, Marzocchi, M: A critical point theory for nonsmooth functionals. Ann. Mat. Pura Appl. 167(4), 73-100 (1994)

10. Ambrosetti, A, Rabinowitz, PH: Dual variational methods in critical point theory and applications. J. Funct. Anal. 14, 349-381 (1973)

11. Silva, EAB: Critical point theorems and applications to differential equations. Ph.D. thesis, University of Wisconsin-Madison (1988)

12. Brasco, L, Franzina, G: On the Hong-Krahn-Szego inequality for the $p$-Laplace operator. Manuscr. Math. 141, 537-557 (2013)

13. Bartsh, T, Wang, ZQ: Existence and multiplicity results for some superlinear elliptic problems on $\mathbb{R}^{N}$. Commun. Partial Differ. Equ. 20, 1725-1741 (1995)

14. Rabinowitz, PH: On a class of nonlinear Schrödinger equations. Z. Angew. Math. Phys. 43, $270-291$ (1992)

15. Katriel, G: Mountain pass theorems and global homeomorphism theorems. Ann. Inst. Henri Poincaré, Anal. Non Linéaire 11, 189-209 (1994)

16. Rabinowitz, PH: Minimax Methods in Critical Point Theory with Applications to Differential Equations. CBMS Regional Conf. Ser. Math., vol. 65. Am. Math. Soc., Providence (1986)

17. Ladyzenskaya, OA, Uralceva, NN: Equations aux dérivées partielles de type elliptiques. Dunod, Paris (1968)

18. Brezis, H, Lieb, E: A relation between pointwise convergence of functions and convergence of functionals. Proc. Am Math. Soc. 88, 486-490 (1983)

19. Boccardo, L, Murat, F: Almost everywhere convergence of the gradients of solutions to elliptic and parabolic equations. Nonlinear Anal. 19, 581-597 (1992)

20. Boccardo, L, Murat, F, Puel, JP: Existence de solutions non bornées pour certaines équations quasi-linéaires. Port. Math. 41, 507-534 (1982)

21. Brezis, H, Browder, FE: Sur une propriété des espaces de Sobolev. C. R. Math. Acad. Sci. Paris 287, 113-115 (1978)

doi:10.1186/1687-2770-2013-179

Cite this article as: Jia et al.: Infinitely many solutions for a class of quasilinear elliptic equations with $p$-Laplacian in $\mathbb{R}^{N}$. Boundary Value Problems 2013 2013:179.

\section{Submit your manuscript to a SpringerOpen ${ }^{\circ}$ journal and benefit from:}

- Convenient online submission

Rigorous peer review

- Immediate publication on acceptance

- Open access: articles freely available online

- High visibility within the field

- Retaining the copyright to your article 